\title{
Rate Acceleration of Anionic Oxy-Cope Rearrangements Induced by an Additional
}

\section{Unsaturation}

Lionel Gentric, Issam Hanna,* Alexandre Huboux, Rachida Zaghdoudi

Laboratoire de Synthèse Organique associé au CNRS, Ecole Polytechnique, F-91128

Palaiseau Cedex, France

\section{Supporting Information}

Characterization data for compounds 8-10, 15-18 and 29-34.

\section{Ketone 8}

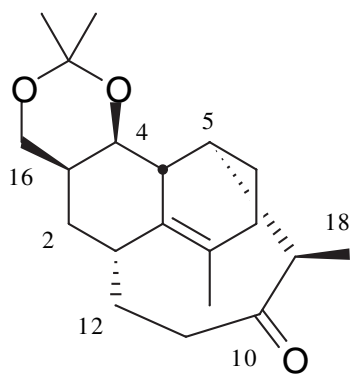

White solid $\mathrm{mp} 131-133^{\circ} \mathrm{C}$ (from $\mathrm{Et}_{2} \mathrm{O} /$ petroleum ether 1:1). Rf 0.20 (15\% EtOAc in petroleum ether). IR $\left(\mathrm{CCl}_{4}\right) v_{\max } 1685 \mathrm{~cm}^{-1} .{ }^{1} \mathrm{H}$ NMR $\left(400 \mathrm{MHz}, \mathrm{CDCl}_{3}\right) \delta 4.34(\mathrm{dd}, J=5.9,2.0 \mathrm{~Hz}, 1 \mathrm{H}, \mathrm{H}-4), 3.91(\mathrm{dd}$, $J=11.5,4.8 \mathrm{~Hz}, 1 \mathrm{H}, \mathrm{H}-16), 3.61(\mathrm{dd}, J=11.5,6.6 \mathrm{~Hz}, 1 \mathrm{H}, \mathrm{H}-16)$, 2.96-2.83 (m, 1H), $2.80(\mathrm{qd}, J=7.0,2.2 \mathrm{~Hz}, 1 \mathrm{H}, \mathrm{H}-9), 2.56-2.36(\mathrm{~m}$, $1 \mathrm{H}, \mathrm{H}-3), 2.35-1.75(\mathrm{~m}, 10 \mathrm{H}), 1.63-1.50(\mathrm{~m}, 1 \mathrm{H}), 1.55(\mathrm{~s}, 3 \mathrm{H}), 1.48(\mathrm{~s}$, $3 \mathrm{H}), 1.36(\mathrm{~s}, 3 \mathrm{H}), 1.23-1.17(\mathrm{~m}, 1 \mathrm{H}), 0.96(\mathrm{~d}, J=6.7 \mathrm{~Hz}, 3 \mathrm{H}, \mathrm{H}-18)$.

${ }^{13} \mathrm{C}$ NMR $\left(100 \mathrm{MHz}, \mathrm{CDCl}_{3}\right) \delta 214.7$ (C, C-10), 133.3 (C, C-8 or C-8a), 132.3 (C, C-8a or C-8), 99.2 (C, acetonide), $69.5(\mathrm{CH}, \mathrm{C}-4), 63.7\left(\mathrm{CH}_{2}, \mathrm{C}-16\right), 50.2(\mathrm{CH}), 44.6(\mathrm{CH}), 43.0$ $\left(\mathrm{CH}_{2}\right), 42.7(\mathrm{CH}), 32.7(\mathrm{CH}), 31.9(\mathrm{CH}), 30.7\left(\mathrm{CH}_{2}\right), 28.4\left(2 \mathrm{CH}_{2}\right), 28.3\left(\mathrm{CH}_{3}\right), 22.2\left(\mathrm{CH}_{3}\right)$, $18.7\left(\mathrm{CH}_{2}\right), 18.7\left(\mathrm{CH}_{3}\right), 16.6\left(\mathrm{CH}_{3}\right) . \mathrm{MS}\left(\mathrm{PCI} / \mathrm{NH}_{3}\right) \mathrm{m} / z 319\left(\mathrm{MH}^{+}\right), 301\left(\mathrm{M}-\mathrm{H}_{2} \mathrm{O}+\mathrm{H}\right)^{+}, 278$ $\left(\mathrm{M}-\left(\mathrm{CH}_{3}\right)_{2} \mathrm{CO}+\mathrm{NH}_{4}\right)^{+}, 261\left(\mathrm{M}-\left(\mathrm{CH}_{3}\right)_{2} \mathrm{CO}+\mathrm{H}\right)^{+}, 243\left(\mathrm{M}-\left(\mathrm{CH}_{3}\right)_{2} \mathrm{CO}-\mathrm{H}_{2} \mathrm{O}+\mathrm{H}\right)^{+}, 225(\mathrm{M}-$ $\left.\left(\mathrm{CH}_{3}\right)_{2} \mathrm{CO}-2 \mathrm{H}_{2} \mathrm{O}+\mathrm{H}\right)^{+}$.

The structure of this compound was confimed by X-ray crystallographic analysis.

\section{Ketone 9.}

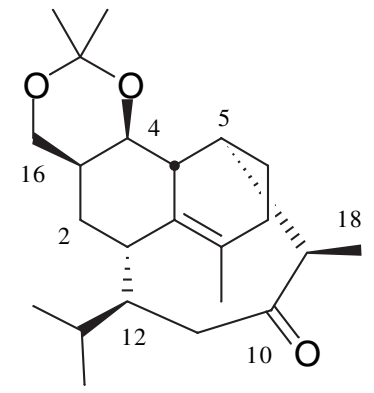

White solid. $\mathrm{R}_{f} 0.35$ (15\% EtOAc in petroleum ether) $\mathrm{mp} 141-145^{\circ} \mathrm{C}$. IR $\left(\mathrm{CCl}_{4}\right) v_{\max } 1681 \mathrm{~cm}^{-1} .{ }^{1} \mathrm{H}$ NMR $\left(400 \mathrm{MHz}, \mathrm{CDCl}_{3}\right) \delta 4.31(\mathrm{dd}$, $J=7.8,5.1 \mathrm{~Hz}, 1 \mathrm{H}, \mathrm{H}-4), 3.67$ (dd, $J=10.8,5.6 \mathrm{~Hz}, 1 \mathrm{H}, \mathrm{H}-16), 3.58$ (t, $J=10.8 \mathrm{~Hz}, 1 \mathrm{H}, \mathrm{H}-16), 2.75$ (dq, $J=6.8,2.0 \mathrm{~Hz}, 1 \mathrm{H}, \mathrm{H}-9), 2.61$ 2.59 (m, 1H, H-1), 2.50-2.39 (m, 1H, H-3), 2.17-2.09 (m, 2H, H-4a, H-5), 1.99-1.84 (m, 6H, H-11, H-11, H-12, H-13, H-6, H-7), 1.77-1.70 (m, 2H, H-2, H-6), 1.64-1.50 (m, 1H, H-7), 1.50 (s, 3H, H-17), 1.45 (s, 3H, acetonide), 1.36 (s, 3H, acetonide), 1.36-1.27 (m, 1H, H-2), 0.98 (d, $J=6.8 \mathrm{~Hz}, 3 \mathrm{H}, \mathrm{PPr}), 0.97$ (d, $J=6.8 \mathrm{~Hz}, 3 \mathrm{H}, \mathrm{iPr}), 0.82$ (d, 
$J=6.8 \mathrm{~Hz}, 3 \mathrm{H}, \mathrm{H}-18) .{ }^{13} \mathrm{C} \mathrm{NMR}\left(100 \mathrm{MHz}, \mathrm{CDCl}_{3}\right) \delta 212.1$ (C, C-10), $134.4(\mathrm{C}), 131.3(\mathrm{C})$, 99.5 (C, acetonide), $68.4(\mathrm{CH}, \mathrm{C}-4), 62.8\left(\mathrm{CH}_{2}, \mathrm{C}-16\right), 49.4(\mathrm{CH}, \mathrm{C}-4 \mathrm{a}), 44.0(\mathrm{CH}, \mathrm{C}-5), 42.1$ $\left(\mathrm{CH}_{2}, \mathrm{C}-11\right), 41.9(\mathrm{CH}, \mathrm{C}-12), 40.7(\mathrm{CH}, \mathrm{C}-9), 34.2(\mathrm{CH}, \mathrm{C}-1), 32.5(\mathrm{CH}, \mathrm{C}-3), 27.4\left(\mathrm{CH}_{2}\right.$, C-7), $27.2\left(\mathrm{CH}_{3}\right.$, acetonide), $26.8(\mathrm{CH}, \mathrm{C}-13), 25.1\left(\mathrm{CH}_{2}, \mathrm{C}-2\right), 23.2\left(\mathrm{CH}_{3}\right.$, acetonide $), 22.6$ $\left(\mathrm{CH}_{3}, \mathrm{iPr}\right), 18.4\left(\mathrm{CH}_{2}, \mathrm{C}-6\right), 18.3\left(\mathrm{CH}_{3}, \mathrm{C}-17\right), 16.3\left(\mathrm{CH}_{3}, \mathrm{C}-18\right), 15.6\left(\mathrm{CH}_{3}, \mathrm{iPr}\right) . \mathrm{MS}$ (PCI/ $\left.\mathrm{NH}_{3}\right) \mathrm{m} / z 378\left(\mathrm{M}+\mathrm{NH}_{4}^{+}\right), 361\left(\mathrm{MH}^{+}\right), 343\left(\mathrm{M}-\mathrm{H}_{2} \mathrm{O}+\mathrm{H}\right)^{+}, 320\left(\mathrm{M}-\left(\mathrm{CH}_{3}\right)_{2} \mathrm{CO}+\mathrm{NH}_{4}\right)^{+}, 303(\mathrm{M}-$ $\left.\left(\mathrm{CH}_{3}\right)_{2} \mathrm{CO}+\mathrm{H}\right)^{+}, 285\left(\mathrm{M}-\left(\mathrm{CH}_{3}\right)_{2} \mathrm{CO}-\mathrm{H}_{2} \mathrm{O}+\mathrm{H}\right)^{+}, 267\left(\mathrm{M}-\left(\mathrm{CH}_{3}\right)_{2} \mathrm{CO}-2 \mathrm{H}_{2} \mathrm{O}+\mathrm{H}\right)^{+}$. Anal. Calcd for $\mathrm{C}_{23} \mathrm{H}_{36} \mathrm{O}_{3}$ : C, 76.62; H, 10.06. Found C, 76.77; H, 10.18.

The structure of this compound was confimed by X-ray crystallographic analysis.

\section{Hydroxyketone 10}

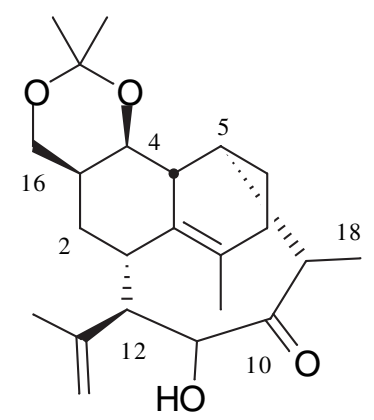

White solid. Rf 0.10 (20\% EtOAc in petroleum ether). IR $\left(\mathrm{CCl}_{4}\right) \mathrm{v}_{\max }$ 3530, 1695, 1455, 1378, 1224, $1115 \mathrm{~cm}^{-1} .{ }^{1} \mathrm{H} \mathrm{NMR}\left(400 \mathrm{MHz}, \mathrm{CDCl}_{3}\right.$ ) $\delta 5.03$ (br s, 1H, H-14), 4.97 (br s, 1H, H-14), 4.37 (dd, $J=7.8,5.2$ $\mathrm{Hz}, 1 \mathrm{H}, \mathrm{H}-4), 3.87$ (d, $J=11.1 \mathrm{~Hz}, 1 \mathrm{H}, \mathrm{H}-11), 3.67$ (dd, $J=10.9,5.6$ $\mathrm{Hz}, 1 \mathrm{H}, \mathrm{H}-16), 3.57$ (dd, $J=10.9,9.5 \mathrm{~Hz}, 1 \mathrm{H}, \mathrm{H}-16), 2.93$ (qd, $J=$ 6.7, $1.9 \mathrm{~Hz}, 1 \mathrm{H}, \mathrm{H}-9), 2.87$ (t, $J=11.4 \mathrm{~Hz}, 1 \mathrm{H}), 2.80$ (dt, $J=11.4,2.8 \mathrm{~Hz}, 1 \mathrm{H}), 2.61-2.49$ (m, 1H), 2.22 (br d, $J=8.2 \mathrm{~Hz}, 1 \mathrm{H}), 2.13$ (br s, 1H), 2.00-1.86 (m, 2H), 1.82-1.77 (m, 1H), 1.80 (s, 3H, H-15), 1.72-1.60 (m, 3H), 1.54 (br s, 3H, H-17), 1.47 (s, 3H, acetonide), 1.36 (s, 3H, acetonide), $1.33-1.23(\mathrm{~m}, 1 \mathrm{H}), 1.08(\mathrm{~d}, J=6.7 \mathrm{~Hz}, 3 \mathrm{H}, \mathrm{H}-18) .{ }^{13} \mathrm{C} \mathrm{NMR}\left(100 \mathrm{MHz}, \mathrm{CDCl}_{3}\right) \delta$ 206.2 (C, C-10), 142.8 (C, C-13), 133.4 (C, C-8 or C-8a), 133.1 (C, C-8a or C-8), $115.3\left(\mathrm{CH}_{2}\right.$, C-14), 99.6 (C, acetonide), 88.9 (CH, C-11), $68.3(\mathrm{CH}, \mathrm{C}-4), 62.8\left(\mathrm{CH}_{2}, \mathrm{C}-16\right), 49.6(\mathrm{CH})$, $48.0(\mathrm{CH}), 44.0(\mathrm{CH}), 40.4(\mathrm{CH}), 31.8(\mathrm{CH}), 31.7(\mathrm{CH}), 27.5\left(\mathrm{CH}_{2}\right), 27.4\left(\mathrm{CH}_{3}\right), 25.4\left(\mathrm{CH}_{2}\right)$, $23.5\left(\mathrm{CH}_{3}\right), 18.6\left(\mathrm{CH}_{3}\right), 18.2\left(\mathrm{CH}_{2}\right), 18.1\left(\mathrm{CH}_{3}\right), 16.2\left(\mathrm{CH}_{3}\right)$. MS $\left(\mathrm{PCI} / \mathrm{NH}_{3}\right) \mathrm{m} / z 375\left(\mathrm{MH}^{+}\right)$, $357\left(\mathrm{M}-\mathrm{H}_{2} \mathrm{O}+\mathrm{H}\right)^{+}, 334\left(\mathrm{M}-\left(\mathrm{CH}_{3}\right)_{2} \mathrm{CO}+\mathrm{NH}_{4}\right)^{+}, 318\left(\mathrm{M}-\left(\mathrm{CH}_{3}\right)_{2} \mathrm{CO}+\mathrm{H}\right)^{+}, 299\left(\mathrm{M}-\left(\mathrm{CH}_{3}\right)_{2} \mathrm{CO}-\right.$ $\left.\mathrm{H}_{2} \mathrm{O}+\mathrm{H}\right)^{+}, 281\left(\mathrm{M}-\left(\mathrm{CH}_{3}\right)_{2} \mathrm{CO}-2 \mathrm{H}_{2} \mathrm{O}+\mathrm{H}\right)^{+}$. 


\section{Hydroxy ketone 15a:}

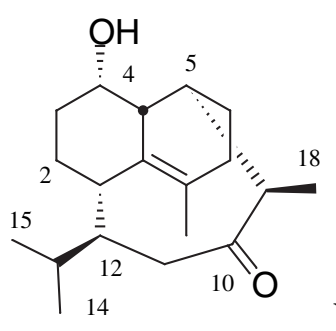

White solid mp 99.5-100 ${ }^{\circ} \mathrm{C}$ (from petroleum ether). IR $\left(\mathrm{CCl}_{4}\right) \mathrm{v}_{\max }$ 3627, $1677 \mathrm{~cm}^{-1}$; ${ }^{1} \mathrm{H}$ NMR (400 MHz, C6 $\left.\mathrm{D}_{6}\right) \delta 3.83\left(\mathrm{~m}, 1 \mathrm{H}, \mathrm{H}_{4}\right), 3.63(\mathrm{qd}, J=6.9$ and 1.6 $\mathrm{Hz}, 1 \mathrm{H}, \mathrm{H}_{9}$ ), 2.75 (dddd, $J=13,11,3.6$ and $2.2 \mathrm{~Hz}, 1 \mathrm{H}, \mathrm{H}_{12}$ ), 2.58 (ddd, $J=11,8.4$ and 3.1 $\left.\mathrm{Hz}, 1 \mathrm{H}, \mathrm{H}_{1}\right), 2.31\left(\mathrm{dt}, J=13\right.$ and $\left.8.0 \mathrm{~Hz}, 1 \mathrm{H}, \mathrm{H}_{7}\right), 2.16\left(\mathrm{br} \mathrm{d}, J=8.4 \mathrm{~Hz}, 1 \mathrm{H}, \mathrm{H}_{5}\right), 2.10$ (dd, $J=17$ and $\left.13 \mathrm{~Hz}, 1 \mathrm{H}, \mathrm{H}_{11}\right), 1.95-1.80\left(\mathrm{~m}, 4 \mathrm{H}, \mathrm{H}_{6}, \mathrm{H}_{7}, \mathrm{H}_{11}\right.$ and $\left.\mathrm{H}_{15}\right), 1.78(\mathrm{br} \mathrm{d}, J=6.4 \mathrm{~Hz}$, $\left.1 \mathrm{H}, \mathrm{H}_{4 \mathrm{a}}\right), 1.66$ (ddd, $J=8.2,6.0$ and $\left.2.9 \mathrm{~Hz}, 1 \mathrm{H}, \mathrm{H}_{2}\right), 1.62\left(\mathrm{~m}, 1 \mathrm{H}, \mathrm{H}_{2}\right), 1.61$ (br s, 3H, $\mathrm{H}_{13}$ ), $1.48\left(\mathrm{~m}, 1 \mathrm{H}, \mathrm{H}_{3}\right), 1.44$ (dddd, $J=14,11,8.5$ and $\left.2.3 \mathrm{~Hz}, 1 \mathrm{H}, \mathrm{H}_{6}\right), 1.21$ (dddd, $J=14,11,8$ and $\left.2.1 \mathrm{~Hz}, 1 \mathrm{H}, \mathrm{H}_{3}\right), 1.10\left(\mathrm{~d}, J=6.8 \mathrm{~Hz}, 1 \mathrm{H}, \mathrm{H}_{14}\right), 0.96$ (br s, $\left.1 \mathrm{H}, \mathrm{OH}\right), 0.89$ (d, $J=7.0 \mathrm{~Hz}$, $3 \mathrm{H}), 0.74(\mathrm{~d}, J=6.9 \mathrm{~Hz}, 3 \mathrm{H}) ;{ }^{13} \mathrm{C} \mathrm{NMR}\left(50 \mathrm{MHz}, \mathrm{CDCl}_{3}\right) \delta 214.5\left(\mathrm{C}_{10}\right), 135.1$ and 133.7 $\left(\mathrm{C}_{8}\right.$ and $\left.\mathrm{C}_{8 \mathrm{a}}\right), 69.0\left(\mathrm{C}_{4}\right), 47.2(\mathrm{CH}), 46.6(\mathrm{CH}), 44.7(\mathrm{CH}), 42.5\left(\mathrm{C}_{11}\right), 40.9(\mathrm{CH}), 32.6(\mathrm{CH})$, $29.7\left(\mathrm{CH}_{2}\right), 28.1(\mathrm{CH}), 27.6\left(\mathrm{CH}_{2}\right), 22.9(\mathrm{Me}), 21.8\left(\mathrm{CH}_{2}\right), 20.2\left(\mathrm{CH}_{2}\right), 18.7(\mathrm{Me}), 16.3(\mathrm{Me})$ and 15.7 (Me). Anal. Calcd for $\mathrm{C}_{19} \mathrm{H}_{30} \mathrm{O}_{2}$ : C, 78.57; H, 10.41. Found C, 78.83; H, 10.45.

The structure of this compound was confimed by X-ray crystallographic analysis.

\section{Hydroxyketone 16.}

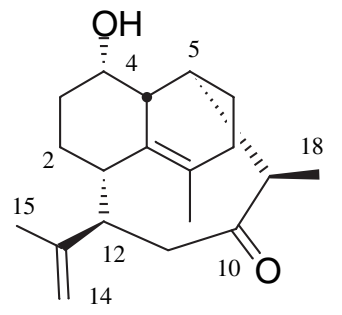

White solid mp $133-135{ }^{\circ} \mathrm{C}$ (EtAOc/petroleum ether). $\mathrm{R}_{f} 0.35$ (10\% EtOAc in petroleum ether) IR $\left(\mathrm{CCl}_{4}\right) \mathrm{v}_{\max } 3624,3490,3071,1679,1639 \mathrm{~cm}^{-1} .{ }^{1} \mathrm{H}$ NMR $\left(400 \mathrm{MHz}, \mathrm{CDCl}_{3}\right) \delta 4.89$ (br s, 1H, H-14), 4.84 (br s, 1H, H-14), 4.39-4.36 (m, 1H,), 3.72 (td, $J=11.2,3.1 \mathrm{~Hz}, 1 \mathrm{H})$, $3.57(\mathrm{qd}, J=6.8,1.8 \mathrm{~Hz}, 1 \mathrm{H}), 2.75(\mathrm{t}, J=9.6 \mathrm{~Hz}, 1 \mathrm{H}), 2.35(\mathrm{~d}, J=8.2 \mathrm{~Hz}, 1 \mathrm{H}), 2.19(\mathrm{dd}, J=$ 17.3, 13.3 Hz, 1H), 2.10-1.70 (m, 10H), 1.70 (s, 3H, H-15), 1.56 (s, 3H, H-17), 1.56-1.47 (m, $1 \mathrm{H}), 0.92(\mathrm{~d}, J=6.8 \mathrm{~Hz}, 3 \mathrm{H}, \mathrm{H}-18) .{ }^{13} \mathrm{C} \mathrm{NMR}\left(100 \mathrm{MHz}, \mathrm{CDCl}_{3}\right) \delta 213.0(\mathrm{C}, \mathrm{C}-10), 147.3(\mathrm{C}$, C-13), 134.3 (C), $134.1(\mathrm{C}), 112.5\left(\mathrm{CH}_{2}, \mathrm{C}-14\right), 69.1(\mathrm{CH}, \mathrm{C}-4), 49.1(\mathrm{CH}), 48.6\left(\mathrm{CH}_{2}\right), 47.2$ 
$(\mathrm{CH}), 44.5(\mathrm{CH}), 40.9(\mathrm{CH}), 32.2(\mathrm{CH}), 29.4\left(\mathrm{CH}_{2}\right), 27.5\left(\mathrm{CH}_{2}\right), 20.5\left(\mathrm{CH}_{2}\right), 20.1\left(\mathrm{CH}_{2}\right), 19.0$ $\left(\mathrm{CH}_{3}\right), 18.6\left(\mathrm{CH}_{3}\right), 16.2\left(\mathrm{CH}_{3}\right)$. MS $\left(\mathrm{PCI} / \mathrm{NH}_{3}\right) \mathrm{m} / z 289\left(\mathrm{MH}^{+}\right), 271\left(\mathrm{M}-\mathrm{H}_{2} \mathrm{O}+\mathrm{H}\right)^{+}$.

\section{Hydroxy ketone 18.}

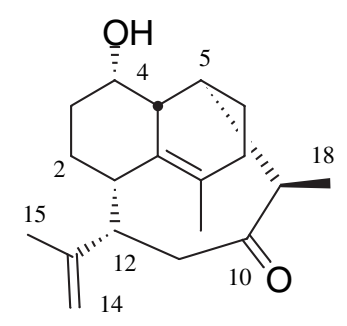

White solid. $\mathrm{R}_{f} 0.30$ (10\% EtOAc in petroleum ether). IR $\left(\mathrm{CCl}_{4}\right) v_{\text {max }}$ 3619, 3082, 1692, $1643 \mathrm{~cm}^{-1} .{ }^{1} \mathrm{H}$ NMR (400 MHz, $\mathrm{CDCl}_{3}$ ) $\delta 4.81$ (s, 1H, H-14), 4.55 (s, 1H, H-14), 4.42-4.37 (m, 1H, H-4), 3.68 (qd, $J=6.8,4.3 \mathrm{~Hz}, 1 \mathrm{H}, \mathrm{H}-9$ ), 3.33 (t, $J=11.9 \mathrm{~Hz}, 1 \mathrm{H}$ ), 3.12-3.05 (m, 1H), 2.98-2.92 (m, 1H), 2.57-2.51 (m, 1H), 2.39-2.34 (m, 1H), $1.97(\mathrm{dd}, J=$ 11.9, $2.3 \mathrm{~Hz}, 1 \mathrm{H}), 1.92-1.73$ (m, 5H), 1.80 (s, 3H, H-15), 1.65-1.57 (m, 1H), 1.63 (s, 3H, H17), 1.44-1.33 (m, 2H), $0.94(\mathrm{~d}, J=6.8 \mathrm{~Hz}, 3 \mathrm{H}, \mathrm{H}-18) .{ }^{13} \mathrm{C} \mathrm{NMR}\left(100 \mathrm{MHz}, \mathrm{CDCl}_{3}\right) \delta 219.2$ (C, C-10), 147.6 (C, C-13), 135.0 and 131.0 (C-8 and C-8a), $110.1\left(\mathrm{CH}_{2}, \mathrm{C}-14\right), 69.0(\mathrm{CH}, \mathrm{C}-$ 4), $50.1(\mathrm{CH}), 47.4(\mathrm{CH}), 44.9(\mathrm{CH}), 44.2\left(\mathrm{CH}_{2}\right), 43.1(\mathrm{CH}), 32.6(\mathrm{CH}), 31.1\left(\mathrm{CH}_{2}\right), 29.5$

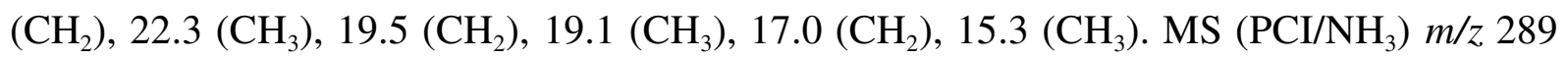
$\left(\mathrm{MH}^{+}\right), 271\left(\mathrm{M}-\mathrm{H}_{2} \mathrm{O}+\mathrm{H}\right)^{+}$.

\section{Compound 29}

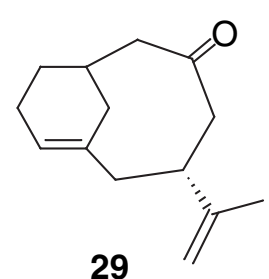

Colorless oil. $\mathrm{R}_{f} 0.40$ (5\% EtOAc in petroleum ether). IR $\left(\mathrm{CCl}_{4}\right) v_{\max } 3081,1697,1644 \mathrm{~cm}^{-1}$. ${ }^{1} \mathrm{H}$ NMR (400 MHz, $\left.\mathrm{CDCl}_{3}\right) \delta 5.51-5.46(\mathrm{~m}, 1 \mathrm{H}), 4.73(\mathrm{~s}, 1 \mathrm{H}), 4.71(\mathrm{~s}, 1 \mathrm{H}), 2.82(\mathrm{t}, J=12$ $\mathrm{Hz}, 1 \mathrm{H}), 2.75(\mathrm{dd}, J=11,7 \mathrm{~Hz}, 1 \mathrm{H}), 2.74-2.69(\mathrm{~m}, 1 \mathrm{H}), 2.62-2.52(\mathrm{~m}, 1 \mathrm{H}), 2.41$ (dd, $J=$ 12.3, $5.2 \mathrm{~Hz}, 1 \mathrm{H}), 2.36$ (br s, 2H), $2.22(\mathrm{~d}, J=10.9 \mathrm{~Hz}, 1 \mathrm{H}), 2.14$ (d, $J=12.3 \mathrm{~Hz}, 1 \mathrm{H}), 2.10-$ $2.02(\mathrm{~m}, 1 \mathrm{H}), 2.01-1.90(\mathrm{~m}, 1 \mathrm{H}), 1.89-1.78(\mathrm{~m}, 2 \mathrm{H}), 1.74(\mathrm{~s}, 3 \mathrm{H}), 1.48-1.60(\mathrm{~m}, 1 \mathrm{H}) .{ }^{13} \mathrm{C}$ NMR (100 MHz, $\left.\mathrm{CDCl}_{3}\right) \delta 215.0(\mathrm{C}), 148.5(\mathrm{C}), 136.4(\mathrm{C}), 127.6(\mathrm{CH}), 108.9\left(\mathrm{CH}_{2}\right), 52.1$ $(\mathrm{CH}), 51.1\left(\mathrm{CH}_{2}\right), 46.0\left(\mathrm{CH}_{2}\right), 40.9\left(\mathrm{CH}_{2}\right), 34.6\left(\mathrm{CH}_{2}\right), 32.8(\mathrm{CH}), 26.3\left(\mathrm{CH}_{2}\right), 22.5\left(\mathrm{CH}_{2}\right)$, $20.4\left(\mathrm{CH}_{3}\right)$. 


\section{Compound 30}

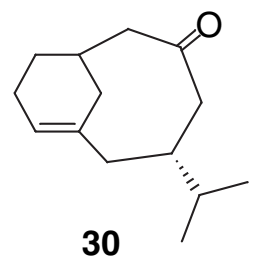

Colorless oil. $\mathrm{R}_{f} 0.30$ (5\% EtOAc in petroleum ether). IR $\left(\mathrm{CCl}_{4}\right) \mathrm{v}_{\max } 1697 \mathrm{~cm}^{-1} .{ }^{1} \mathrm{H} \mathrm{NMR}$ $\left(400 \mathrm{MHz}, \mathrm{CDCl}_{3}\right) \delta 5.50-5.38(\mathrm{~m}, 1 \mathrm{H}), 2.74(\mathrm{dd}, J=11,7 \mathrm{~Hz}, 1 \mathrm{H}), 2.62(\mathrm{t}, J=12 \mathrm{~Hz}, 1 \mathrm{H})$, 2.60-2.46 (m, 1H), 2.39-2.15 (m, 4H), 2.10-1.90 (m, 4H), 1.80 (dd, J = 14, 7 Hz, 1H), 1.79$1.45(\mathrm{~m}, 3 \mathrm{H}), 0.91(\mathrm{~d}, J=4.5 \mathrm{~Hz}, 3 \mathrm{H}), 0.89$ (d, $J=4.5 \mathrm{~Hz}, 3 \mathrm{H}) . \mathrm{MS}$ (EI ) $m / z 206\left(\mathrm{M}^{+}\right)$.

\section{Compound 33}<smiles>C=C(C)[C@H]1CC(=O)CC2CCC=C[C@@H]21</smiles>

33 Colorless oil. $\mathrm{R}_{f} 0.40$ (5\% EtOAc in petroleum ether). IR $\left(\mathrm{CCl}_{4}\right) \mathrm{v}_{\max } 3086$, 3028, 1705, $1644 \mathrm{~cm}^{-1} .{ }^{1} \mathrm{H}$ NMR (400 MHz, $\left.\mathrm{CDCl}_{3}\right) \delta 6.10-5.95(\mathrm{~m}, 2 \mathrm{H}), 4.87(\mathrm{~s}, 1 \mathrm{H}), 4.84(\mathrm{~s}$, $1 \mathrm{H}), 2.96(\mathrm{t}, J=10.6 \mathrm{~Hz}, 1 \mathrm{H}), 2.72-2.62(\mathrm{~m}, 2 \mathrm{H}), 2.62-2.52(\mathrm{~m}, 2 \mathrm{H}), 2.40-2.25(\mathrm{~m}, 2 \mathrm{H}), 2.25-$ $2.10(\mathrm{~m}, 1 \mathrm{H}), 2.10-2.00(\mathrm{~m}, 1 \mathrm{H}), 1.90-1.80(\mathrm{~m}, 1 \mathrm{H}), 1.77(\mathrm{~s}, 3 \mathrm{H}), 1.75-1.55(\mathrm{~m}, 2 \mathrm{H}) .{ }^{13} \mathrm{C}$ NMR (100 MHz, $\left.\mathrm{CDCl}_{3}\right) \delta 214.0(\mathrm{C}), 147.1(\mathrm{C}), 133.4(\mathrm{CH}), 132.1(\mathrm{CH}), 110.7\left(\mathrm{CH}_{2}\right), 55.3$ $\left(\mathrm{CH}_{2}\right), 45.5(\mathrm{CH}), 43.0\left(\mathrm{CH}_{2}\right), 34.0(\mathrm{CH}), 29.1(\mathrm{CH}), 27.3\left(\mathrm{CH}_{2}\right), 23.0\left(\mathrm{CH}_{3}\right), 19.4\left(\mathrm{CH}_{2}\right) . \mathrm{MS}$ $\left(\mathrm{PCI} / \mathrm{NH}_{3}\right) \mathrm{m} / z 191\left(\mathrm{MH}^{+}\right), 208\left(\mathrm{M}+\mathrm{NH}_{4}\right)^{+}$.

\section{Compound 34}<smiles>C=C(C)C1CC(=O)CC2CCC=CC21</smiles>

Colorless oil. $\mathrm{R}_{f} 0.50$ (5\% EtOAc in petroleum ether). IR $\left(\mathrm{CCl}_{4}\right) \mathrm{v}_{\max } 3088$, 3024, 1718, $1646 \mathrm{~cm}^{-1} .{ }^{1} \mathrm{H}$ NMR (400 MHz, $\left.\mathrm{CDCl}_{3}\right) \delta 5.78-5.88(\mathrm{~m}, 1 \mathrm{H}), 5.50$ (br d, $J=10.6$ $\mathrm{Hz}, 1 \mathrm{H}), 4.97$ (s, 1H), 4.62 (s, 1H), 2.73 (br s, 1H), 2.52-2.22 (m, 4H), 2.25 (br d, J = $12 \mathrm{~Hz}$, $1 \mathrm{H}), 2.18-2.08(\mathrm{~m}, 3 \mathrm{H}), 1.95-1.85(\mathrm{~m}, 1 \mathrm{H}), 1.79(\mathrm{~s}, 3 \mathrm{H}), 1.70-1.60(\mathrm{~m}, 1 \mathrm{H}) .{ }^{13} \mathrm{C}$ NMR $(100$ $\left.\mathrm{MHz}, \mathrm{CDCl}_{3}\right) \delta 212.8(\mathrm{C}), 144.7(\mathrm{C}), 129.0(\mathrm{CH}), 124.0(\mathrm{CH}), 111.1\left(\mathrm{CH}_{2}\right), 46.5(\mathrm{CH}), 41.2$ $\left(\mathrm{CH}_{2}\right), 40.9\left(\mathrm{CH}_{2}\right), 35.5(2 \mathrm{CH}), 26.8\left(\mathrm{CH}_{2}\right), 22.6\left(\mathrm{CH}_{3}\right), 20.9\left(\mathrm{CH}_{2}\right) . \mathrm{MS}\left(\mathrm{PCI} / \mathrm{NH}_{3}\right) \mathrm{m} / z, 191$ $\left(\mathrm{MH}^{+}\right), 208\left(\mathrm{M}+\mathrm{NH}_{4}\right)^{+}$. 